\title{
Sun-Climate Complexity Linking
}

\author{
B. J. West ${ }^{1,2}$ and P. Grigolini ${ }^{3,4,5}$ \\ ${ }^{1}$ Mathematical \& Information Science, Army Research Office, Research Triangle Park, North Carolina 27709, USA \\ ${ }^{2}$ Physics Department, Duke University, Durham, North Carolina 27705, USA \\ ${ }^{3}$ Center for Nonlinear Science, University of North Texas, Denton, Texas 76203, USA \\ ${ }^{4}$ Istituto del Processi Chimico Fisici del CNR, Area della Ricera di Pisa, Pisa, Italy \\ ${ }^{5}$ Dipartimento di Fisica dell'Universitá di Pisa, Largo Pontecorvo 3, 56127 Pisa, Itay
}

(Received 11 July 2007; published 29 February 2008)

\begin{abstract}
It is known that Earth's short-term temperature anomalies share the same complexity index $\mu$ as solar flares. We show that this property is not accidental and is a consequence of the phenomenon of information transfer based on the crucial role of non-Poisson renewal events in complex networks.
\end{abstract}

A cornerstone of statistical physics is the fluctuationdissipation theorem (FDT) of the first kind and the linear response theory (LRT) of Kubo [1] on which it rests. Recently, with the increasing importance of phenomena whose statistics do not satisfy the central limit theorem, a special version of the FDT was developed to study the response of renewal non-Poisson networks to external perturbations. This theory is a kind of generalization of the well-known theory of stochastic resonance (SR) proposed in 1981 to explain the periodic recurrence of ice ages [2]. The current generalization is called the event dominated fluctuation-dissipation theorem (EDFDT) of the first kind $[3,4]$ and leads to the remarkable result that under certain conditions a complex network being perturbed by a second complex network takes on the statistical properties of the perturbation. This is the complexity matching (CM) phenomenon recently proposed by the authors of Ref. [5] involving the transfer of information, rather than energy, and can be realized with a perturbative linking of extremely weak intensity [4]. CM is expected to apply to all processes dominated by crucial renewal events, as has been shown in the case of earthquakes [6], blinking quantum dots [3], and brain dynamics [7]. Herein we apply this theory to the linking of Earth's climate to total solar irradiance (TSI). The average global temperature is a consequence of the TSI being absorbed and redistributed by Earth's atmosphere and oceans by means of nonlinear hydrothermal dynamic processes [8]. In the past two decades this phenomenon has been framed as a large-scale numerical simulation incorporating all identified physical or chemical mechanisms in an attempt to recreate and understand the variability in Earth's temperature time series [9]. What is not addressed in these simulations, over and above the temperature increase of the past 30 years, are the statistics of the average global temperature and the reasons why the statistics of the temperature anomalies are not Poisson, Gaussian, or any of the other elementary statistical distributions $[10,11]$.

Earth's surface temperature responds to variations in the TSI, which can be partitioned into low-frequency quasi- cycles and the more high-frequency random fluctuations. The low amplitude, approximately 11-year solar cycle influence on climate is well studied, leading to, for example, the decadal oscillation in the stratosphere and troposphere, as well as the resonant excitation of La Nina [12]. On the other hand, on the basis of large-scale computer simulations (see, for example, [13]), the highfrequency random fluctuations in solar activity have been found to be negligible. Consequently, the statistical variability in Earth's surface temperature is interpreted as noise. As noise these fluctuations are thought to contain no useful information and are smoothed to emphasize the presumably more important long-time variations in the average global temperature. However, from nonequilibrium statistical physics we know that statistical fluctuations often provide information about the transport properties of complex phenomena [14].

The general context for reframing global warming is nonequilibrium statistical physics where the coarsegrained meteorological equations used to describe climate change are stochastic, dissipative, and nonlinear. The basis for stochastic climate models was the Langevin equation introduced into climate modeling over three decades ago by Hasselman [15], Leith [16], and the generalized Langevin equation introduced by Lindenberg and West [17] and more recently taken up by Majda et al. [18]. In Hasselman's model of oceanic temperature there is linear damping and the atmosphere provides a stochastic forcing, so that for a "white" noise driver in this simple linear model there is a "red" noise response of the oceanic temperature. The network is not considered to be thermodynamically closed, however, so there is no FDT.

Herein we implement a generalization of Hasselmann's model; one that is thermodynamically closed, that is, the linear damping is tied to the fluctuations through a FDT of the first kind [14]. The use of the term "dissipation" is unfortunate in a geophysical context because dissipation is not a physical quantity such as viscosity, but rather it describes the extraction and redistribution of energy through the internal nonlinear dynamics of Earth's ocean- 
land-atmosphere network. This redistribution is balanced against the stochastic driver through a FDT as we discuss below.

We show herein that the stochastic properties of the average global temperature are linked to the TSI variability and it is this complexity linking within the Sun-climate network that forces Earth's temperature anomalies to inherit the statistics of Sun's activity. Consequently, both TSI and global temperature are observed to have inverse power-law statistical distributions, with the same parameter values, even though the cross-correlation of the random fluctuations vanish except at the lowest frequencies [10,11] where the quasiperiodic solar cycles dominate the dynamics. The variations in the TSI are produced by Sun's turbulent dynamics, as evidenced by changes in solar flare and sunspot activity, as well as strong erratic fluctuations associated with the intermittency [19-22] of those activities. This time variation in TSI induces similar changes in Earth's average temperature, as well as producing trends that move the global temperature up or down for tens or even hundreds of years $[8,19]$. In addition to Sun's erratic dynamics, Earth's atmosphere and oceans are also turbulent so that short-term (days and weeks) changes in global temperature are not directly tied to changes in TSI. Moreover, measures such as the cross-correlation function do not reveal any connection between the short-term temperature fluctuations and the intermittent solar activity.

The authors of Ref. [20] focused their attention on solar flares and reached the conclusion that the sequence $\left\{t_{j}\right\}$, where $t_{j}$ denotes the time of occurrence of the $j$ th solar flare, is a non-Poisson renewal process, whose time asymptotic properties are the same as those generated by the distribution density

$$
\psi_{I}(\tau)=\left(\mu_{I}-1\right) \frac{T_{I}^{\mu_{I}-1}}{\left(T_{I}+\tau\right)^{\mu_{I}}}
$$

with

$$
\mu_{I}=2.138 \pm 0.01 \text {. }
$$

These authors, shuffling the data points and arriving at the same distribution, argued that the solar flares constitute a renewal process that probably reflects the turbulent nature of Sun's internal dynamics. They based their analysis on the diffusion entropy (DE) technique [23,24].

More recently, the authors of Refs. [10,11], also using the DE technique, found that the air temperature anomalies reveal the same complexity as that of solar flares, with a complexity index $\mu_{E}$, which is remarkably close to that of Eq. (2). In fact, they found that the power-law index for the air temperature was $\mu_{E}=2.18$ for the Northern Hemisphere, $\mu_{E}=2.1$ for the Southern Hemisphere, $\mu_{E}=2.19$ over land, and $\mu_{E}=2.07$ over the ocean. In this Letter we argue that this surprising similarity is not accidental, but is a natural consequence of the phenomenon of CM [5].
To concisely review the theory of Refs. [3,4], let us consider a network $S$, which, in the absence of perturbation, would generate the fluctuation $\xi_{S}(t)$. Let us assume that this network is perturbed by a signal $\xi_{P}(t)$, produced by a network $P$. Note that there is no feedback of the network $S$ on $P$. According to Refs. [3,4], when we switch on the linking of $S$ to $P$, we obtain

$$
\left\langle\xi_{S}(t)\right\rangle=\epsilon \int_{0}^{t} \chi_{S}\left(t, t^{\prime}\right) \xi_{P}\left(t^{\prime}\right) d t^{\prime} .
$$

Note that this is the typical form shared by ordinary SR [25] and Kubo LRT [1]. This equation is based on the assumption that we apply the same perturbation to infinitely many identical copies of $S$ and then perform the average over this ensemble, indicated by $\left\langle\xi_{S}(t)\right\rangle$, on the corresponding infinitely many responses. The parameter $\epsilon$ indicates the perturbation strength, and it is assumed to be sufficiently small to ensure the linear response of the network $S$. The key ingredients of the theory of Refs. [3,4] is given by the action of the non-Poisson renewal events, with complexity index $\mu_{S}$, embedded in the fluctuations $\xi_{S}(t)$. The importance of these events has been stressed in [26], showing that for $\mu_{S}<3$ the long-time statistical properties of the network $S$ are determined by these events, in spite of camouflage action exerted by the cloud of secondary events, within which these events are imbedded. Following the authors of Ref. [26], we refer to these events as crucial events and we adopt the EDFDT [3,4], yielding

$$
\chi_{S}\left(t, t^{\prime}\right)=-\frac{d}{d t} \Psi_{S}\left(t, t^{\prime}\right),
$$

where $\Psi_{S}\left(t, t^{\prime}\right)$ is the probability that no $S$ crucial event occurs up to time $t$, with the waiting time process beginning at time $t^{\prime}$. Note that the transmission of information from $P$ to $S$ is a consequence of $\xi_{P}\left(t^{\prime}\right)$ slightly perturbing the time of occurrence of the $S$ events. In the spirit of Kubo LRT the effect of this perturbation is expressed in terms of the unperturbed property $\Psi_{S}\left(t, t^{\prime}\right)$. Note that the $S$ ensemble is assumed to be prepared at a time $t=-t_{a}$ : all the systems of this ensemble share the property of a crucial event occurring at the preparation time and, consequently, $t_{a}$ is the ensemble age. If $t_{a}=0$, the survival probability $\Psi_{S}\left(t, t^{\prime}\right)$ violates the stationary condition $\Psi\left(t, t^{\prime}\right)=\Psi\left(t-t^{\prime}\right)$. When $\mu_{S}<\infty$, the only possible way to recover the stationary condition is to assume $t_{a}=$ $-\infty$. In this case [27]

$$
\Psi_{S}\left(t, t^{\prime}\right)=\left(\frac{T_{S}}{T_{S}+t-t^{\prime}}\right)^{\mu_{S}-2},
$$

and, using Eq. (4), we obtain the response function

$$
\chi_{S}\left(t, t^{\prime}\right)=\left(\mu_{S}-2\right) \frac{T^{\mu_{S}-2}}{\left(T+t-t^{\prime}\right)^{\mu_{S}-1}} .
$$

From a formal point of view the assumption $t_{a}=-\infty$ makes the EDFDT coincide with the ordinary FDT (of the first kind) used in the traditionary theory of SR [25], where, however, no attention was given to crucial events. 
In the case of the Sun-climate linking the network $S$ is Earth. Thus, we replace the symbol $S$ with $E$, and make the assumption that the unperturbed fluctuation $\xi_{E}(t)$ is given by $\Delta T(t)$, namely, the fluctuation around a prescribed mean value whose time dependence is not of interest here. We also make the assumption that, in the absence of the action of solar flares (acting as surrogates for the TSI), the crucial events imbedded in the temperature fluctuations are characterized by the power-law index $\mu_{E}^{(0)}$, which is unknown to us. We assume that $\mu_{E}^{(0)}>2$ so as to use the linear response function of Eq. (6), with $\mu_{S}=\mu_{E}^{(0)}$. The superscript (0) emphasizes that this is the complexity index that the DE technique would detect in the absence of the influence of solar flares. The perturbation $\xi_{P}(t)$ is replaced by $\Delta I(t)$, which refers to the fluctuations around the mean value (on the time scale of years) of the TSI. In this case the crucial events are identified with the surrogate data of the solar flares, thereby making $\mu_{P}=\mu_{I}=2.138$, the value of Eq. (2) established in Ref. [20]. This is in keeping with the argument that the time scales of climate and weather play the role of "macroscopic" and "microscopic" time scale, respectively [14].

To realize the purpose of this Letter, we have to illustrate the two main properties on which the DE technique is based. The first property is the adoption of a mobile window of changing size $l$, recording the sequence $\Delta T(t)$ under the influence of Sun's perturbation from $t_{\text {running }}$ to $t_{\text {running }}+l$, with $t_{\text {running }}$ moving along the whole sequence so as to create a Gibbs ensemble: each system of this ensemble is a subsequence of length $l$. As a consequence, the DE technique affords information on $\langle\Delta T(l)\rangle$, making it play the role of $\left\langle\xi_{S}(t)\right\rangle$ in Eq. (3). The maximum transmission of information is realized by the windows with $t_{\text {running }}$ coinciding with the occurrence of a crucial event in $\Delta I(t)$. In fact, there are many values of $t_{\text {running }}$ meeting this condition, and we construct an average over all of them thereby obtaining from Eq. (3)

$$
E(t) \equiv\langle\langle\Delta T(t)\rangle\rangle=\epsilon \int_{0}^{t} \chi_{E}\left(t, t^{\prime}\right) \Psi_{I}\left(t^{\prime}\right) d t^{\prime},
$$

with

$$
\Psi_{I}(t) \equiv\langle\Delta I(t)\rangle \propto\left[\frac{T_{I}}{T_{I}+t}\right]^{\mu_{I}-1},
$$

namely, the probability that no new solar flare occurs up to time $t$, given the condition that the last one occurred at time $t=0$ [27]. Let us insert Eqs. (6) and (8) into Eq. (7) and assume that $\mu_{E}<3$. Taking the Laplace transform of Eq. (7), $\tilde{f}(u) \equiv \int_{0}^{\infty} e^{-u t} f(t) d t$, using the convolution theorem, and taking the limit $u \rightarrow 0$, we obtain

$$
\tilde{E}(u)=\epsilon \frac{T_{I}}{\mu_{I}-2} \tilde{\Psi}_{I}(u) \tilde{\chi}_{E}(u),
$$

with $\left(\mu^{(0)}<2\right)$

$$
\tilde{\chi}_{E}(u)=1-\Gamma\left(3-\mu_{E}^{(0)}\right)\left(T_{E} u\right)^{\mu_{E}^{(0)}-2}+\ldots
$$

and

$$
\tilde{\Psi}_{I}(u)=1-\Gamma\left(3-\mu_{I}\right)\left(T_{I} u\right)^{\mu_{I}-2}+\ldots
$$

Under the condition that the unperturbed power-law index for temperature exceeds that for the TSI

$$
\mu_{E}^{(0)}>\mu_{I}
$$

the asymptotic limit of Eq. (9) becomes

$$
\tilde{E}(u) \approx \epsilon \frac{T_{I}}{\mu_{I}-2}\left[1-\Gamma\left(3-\mu_{I}\right)\left(T_{I} u\right)^{\mu_{I}-2}\right],
$$

thereby implying that $E$ inherits the complexity of the perturbation $I$. The result does not change if $\mu_{E}^{(0)}>2$, insofar as for $u \rightarrow 0, \tilde{\chi}_{E}(u)=1-\frac{T_{E}}{\mu_{E}^{(0)}-1} u+\ldots$ Therefore we conclude that $E$ inherits the complexity of $I$ under the condition Eq. (12) [28].

We now turn our attention to the second property of the DE technique. This is the conversion of the Gibbs ensemble realized by the moving window of size $l$ into a distribution density $p(x, l)$ so as to determine the Shannon entropy

$$
S(l)=-\int_{0}^{\infty} d x p(x, l) \ln p(x, l) .
$$

The presence of crucial events [24,26] makes the probability distribution density $p(x, l)$ satisfy the scaling condition

$$
p(x, l)=l^{-\delta} F\left(\frac{x}{l^{\delta}}\right),
$$

where $F(y)$ is a function of the dimensionless variable $y$, determined by the generalized central limit theorem [24], fitting the normalization condition $\int_{0}^{\infty} d y F(y)=1$, with the scaling coefficient

$$
\delta=\frac{1}{\mu_{E}-1} .
$$

By inserting Eq. (15) into Eq. (14) and using the normalization condition, we obtain

$$
S(l)=A+\delta \ln l,
$$

where $A=-\int_{\infty}^{\infty} d y F(y) \ln F(y)$ is a constant. Thus, the slope of $S(l)$ in a linear-log representation is the scaling coefficient $\delta$. Note that the entropy $S(t)$ does not require the variance of the probability density function to be finite [29], for example, in $\alpha$-stable Lévy processes $(\delta=1 / \alpha)$ all moments $\nu>\alpha$ diverge for $\alpha<2$, and is a Gauss distribution with all moments finite for $\alpha=2$. The existence of scaling in a process with a diverging second moment implies that DE is complementary to and not simply an alternative to other techniques that rely on the central moment properties of the distribution. So, the scaling exponent $\delta$ is conceptually different from the Hurst exponent 
$H$ and suggests that the scaling exponents $\delta$ and $H$ fulfill multiple relations according to the nature of the process under study. One such relation is the Lévy-walk diffusion relation [29] resulting from a Lévy random walk [30]: $0.5<\delta=\frac{1}{3-2 H}=\frac{1}{\mu-1}<1$. The authors of Refs. [10,11] determined that air temperature anomalous fluctuations fit this relation remarkably well, thereby confirming the renewal nature of the crucial events imbedded with $\Delta T(t)$.

For Eq. (3) to apply, the linking strength $\epsilon$ must be extremely small. As a consequence, only a few of the $E$ events inherit the $I$ statistics. However, the few that do so, thanks to Eqs. (12) and (16), generate a larger scaling thereby determining the asymptotic scaling detected by the DE technique, a condition similar to that of Ref. [26]. In the case of air temperature anomalies the role of ordinary events is played by the $E$ events still obeying the unperturbed complexity index $\mu_{E}^{(0)}$, which remains unknown as a consequence of the fact that selecting the time scale of the solar flares has the effect of producing a fast transition to the time asymptotic regime dominated by Sun's crucial events.

To recapitulate, we have shown in this Letter that Earth's air temperature anomalies inherit Sun's complexity by means of the recently discovered CM effect $[4,5]$. The $\mathrm{CM}$ effect is a transfer of information and can be realized through a linking of extremely small intensity, which is, in fact, the condition on which the key prescription of Eq. (3) rests. As mentioned in the Introduction, this phenomenon is a general property of information transmission from $P$ to $S$, thanks to the role of crucial events: the influence of music on the human brain [7] is explained in a similar manner.

P.G. acknowledges financial support from ARO and Welch through Grants No. W911NF-05-1-0205 and No. B-1577, respectively.

[1] R. Kubo, J. Phys. Soc. Jpn. 12, 570 (1957).

[2] R. Benzi, A. Sutera, and A. Vulpiani, J. Phys. A 14, L453 (1981).

[3] G. Aquino, P. Grigolini, and B. J. West, Europhys. Lett. 80, 10002 (2007).

[4] P. Allegrini, M. Bologna, P. Grigolini, and B. J. West, Phys. Rev. Lett. 99, 010603 (2007).

[5] P. Allegrini et al., arXiv:cond-mat/0608341; P. Allegrini et al., arXiv:cond-mat/0612303.

[6] M. S. Mega, P. Allegrini, P. Grigolini, V. Latora, L. Palatella, A. Rapisarda, and S. Vinciguerra, Phys. Rev. Lett. 90, 188501 (2003).

[7] S. Bianco, M. Ignaccolo, M. S. Rider, M. J. Ross, P. Winsor, and P. Grigolini, Phys. Rev. E 75, 061911 (2007).
[8] W. Soon, S. Baliunas, E. S. Posmentier, and P. Okeke, New Astron. Rev. 4, 563 (2000); M. Sharma, Earth Planet. Sci. Lett. 199, 459 (2002); M. Lockwood, J. Geophys. Res. 106, 16021 (2001); W. Soon, S. Baliunas, E. S. Posmentier, and P. Okeke, Ann. Geophys. Hydro. Space Sci. 18, 583 (2000).

[9] M.E. Mann, R.S. Bradley, and M. K. Hughes, Nature (London) 392, 779 (1998).

[10] N. Scafetta and B. J. West, Phys. Rev. Lett. 90, 248701 (2003).

[11] N. Scafetta, P. Grigolini, T. Imholt, J. Roberts, and B. J. West, Phys. Rev. E 69, 026303 (2004).

[12] H. van Loon, G. A. Meehl, and D. J. Shea, J. Geophys. Res. 112, D02108 (2007).

[13] 2007 Intergovernmental Panel on Climate Change (IPCC) Summary for Polycimakersat http://www.ipcc.ch.

[14] K. Lindenberg and B. J. West, The Nonequilibrium Statistical Mechanics of Open and Closed Systems (VCH Publishers, New York, 1990).

[15] K. Hasselman, Tellus 28, 473 (1976).

[16] C. E. Leith, J. Atmos. Sci. 32, 2022 (1975).

[17] K. Lindenberg and B. J. West, J. Atmos. Sci. 41, 3021 (1984).

[18] A. J. Majda, I. Timofeyev, and E. V. Eijnden, Proc. Natl. Acad. Sci. U.S.A. 96, 14687 (1999).

[19] J.D. Fix, Astronomy, Journey to the Cosmic Frontier (McGraw-Hill, New York, 2001), 2nd ed.

[20] P. Grigolini, D. Leddon, and N. Scafetta, Phys. Rev. E 65, 046203 (2002).

[21] G. Boffetta, V. Carbone, P. Giuliani, P. Veltri, and A. Vulpiani, Phys. Rev. Lett. 83, 4662 (1999).

[22] P. Giuliani, V. Carbone, P. Veltri, G. Boffetta, and A. Vulpiani, Physica (Amsterdam) 280A, 75 (2000).

[23] N. Scafetta, P. Hamilton, and P. Grigolini, Fractals 9, 193 (2001).

[24] P. Grigoloini, L. Palatella, and G. Raffaelli, Fractals 9, 439 (2001).

[25] L. Gammaitoni, P. Hanggi, P. Jung, and F. Marchesoni, Rev. Mod. Phys. 70, 223 (1998).

[26] P. Allegrini, F. Barbi, P. Grigolini, and P. Paradisi, Phys. Rev. E 73, 046136 (2006).

[27] P. Allegrini, G. Aquino, P. Grigolini, L. Palatella, A. Rosa, and B. J. West, Phys. Rev. E 71, 066109 (2005).

[28] Note that the theoretical treatment used herein rests on the assumption the perturbation is brand new. If we assume that it is infinitely aged, the theoretical prescriptions of Ref. [27], which was used to obtain Eq. (12), lead to $\mu_{E}^{(0)}>\mu_{I}+1$. This inequality would make the range of attraction of $I$ even more extended, since the case $2<$ $\mu_{E}^{(0)}<\mu_{I}=2.138$ would be attracted by Sun's complexity.

[29] N. Scafetta and P. Grigolini, Phys. Rev. E 66, 036130 (2002).

[30] M. F. Shlesinger, B. J. West, and J. Klafter, Phys. Rev. Lett. 58, 1100 (1987). 